\title{
DEVELOPMENT AND APPLICATION OF THE TRAPEZOIDAL MODEL FOR ARCHAEOLOGICAL CHRONOLOGIES
}

\author{
Sharen Lee ${ }^{1}$ Christopher Bronk Ramsey \\ Oxford Radiocarbon Accelerator Unit (ORAU), Research Laboratory for Archaeology and the History of Art (RLAHA), Uni- \\ versity of Oxford, Dyson Perrins Building, South Parks Road, Oxford OX1 3QY, United Kingdom.
}

\begin{abstract}
Single- and multiphase models have been used extensively in construction of chronologies. We model more gradual transition between phases with a trapezoid model since it better reflects the nature of the information that goes into the model prior. We find that a simple trapezoid model has a bias that does not reflect prior knowledge, and thus propose an addition of a noninformative element to the prior. We also present an alternative parameterization, which transforms the current abrupt transition model into a model that allows for gradual changes. The addition of a noninformative prior ensures model flexibility. We evaluate these Bayesian models using 2 case studies.
\end{abstract}

\section{INTRODUCTION}

Over the recent decades, the advances in computing power, calibration, and freely available analysis software such as OxCal (Bronk Ramsey 1995, 2001, 2009) and BCal (Buck et al. 1999), Bayesian methods have become popular among archaeologists and other researchers wishing to build chronologies. Bayesian statistics provide a tool for combining results from absolute dating with independent relative information found in archaeological contexts. The outputs provide us with more precise and relevant archaeological information than absolute dates alone (e.g. temporal constraints to a group or groups of events).

Phase models are used widely to assign relationships to groups of events. This relationship can be usage, deposition, or production rate of materials in a culture. They have been defined with different mathematical functions (Bronk Ramsey 2009), but the most often used is the uniform prior model (Buck et al. 1992), which assumes a uniform distribution of materials within 2 boundaries. Examples of application of the uniform prior model can be seen in Blackwell and Buck (2003), Buck and Bard (2007), and Blockley et al. (2004). Recent literature discusses the use of a flexible model in the shape of a trapezoid for the construction of more robust chronologies (Housley et al. 1997; Zeidler et al. 1998; Van Strydonck et al. 2004). Application of this model was further discussed by Blackwell and Buck (2003) and Buck and Bard (2007) and implemented in Karlsberg (2006). The trapezoid model is separated into 3 parts: first, a gradual increase (introductory period); then, a period of constant rate of activity (blooming period); and finally, a gradual decrease (period of decline).

This paper discusses the development and application of the trapezoid prior for modeling archaeological chronologies. In this section, we address a simple archaeological problem with the trapezoid model and propose an alternative parameterization. In Section 2, we discuss model limitations and suggest solutions. In Section 3, we evaluate the modifications, and in Section 4, we explain how the new approach works for a multiphase scenario from the literature.

\subsection{Case Study}

Consider a single-phase scenario: Brindley (2007: Chapter 4) reported 72 radiocarbon determinations from 49 graves in Ireland containing food vessel bowl pottery. Bayliss and O'Sullivan (forthcoming) use a uniform prior model to estimate the temporal constraints of the bowl pottery and the associated burial tradition after reassessing the reliability of each ${ }^{14} \mathrm{C}$ date. Bayliss and O'Sullivan (forthcoming) also mention that the trapezoid prior model would be more appropriate for modeling the occurrence of archaeological types. This is because "each type originates at a given time in a

${ }^{1}$ Corresponding author. Email: sharen.lee@rlaha.ox.ac.uk. 
given place, is made gradually in increasing numbers as time goes on, then decreases in popularity until it becomes forgotten, never to reoccur in an identical form" (Brainerd 1951:304).

The temporal constraints that we are interested in are the start and end of the Irish bowl usage. These dates are particularly problematic to estimate because it is extremely unlikely that the physical remains of the very first or the very last artifacts are found in a the same region. Thus, one cannot deduce from ${ }^{14} \mathrm{C}$ dates alone which single date represents the earliest or latest in the period of interest. It is widely known that calibration is one of the first steps in studying and analyzing ${ }^{14} \mathrm{C}$ data. Calibrated ${ }^{14} \mathrm{C}$ ages tend to spread across centuries and overlap each other due to the nonmonotonic nature of the calibration curve (Reimer et al. 2009). It is impossible to accurately determine the precise earliest or latest age, even if those samples are obtained. Using a Bayesian framework, inference can be drawn on the earliest or latest date with information from the ${ }^{14} \mathrm{C}$ material deposited between them. Using the interpretation of Brainerd (1951:304), we assume that the currency of artifact types takes the shape of a trapezoid.

\subsection{Data}

Seventy-two ${ }^{14} \mathrm{C}$ determinations were reported in a catalog produced by Brindley (2007: Chapter 4). Five of these measurements are duplicates used to cross-check the reproducibility using both conventional and accelerator mass spectrometry (AMS) techniques. The conventional measurements (GrN-14616, -11352, -12950, -9323, and -11901) are reported as being too old for reasons unknown, and will be excluded in this analysis. Another 3 duplicate dates are used in a testing program for carbonate from unburnt bone. The carbonate results from these samples (GrA-13331, GrA-13332, and GrA-13330) appear reliable, but carbonate in unburnt bone is likely to take up contamination (Brindley 2007). Thus, these samples are also excluded from this analysis. A further 10 dates (GrN11363, UB-3987, UB-3988, UB-3989, GrN-12958, UB-3985, UB-3586, GrA-24152, GrA-24193, GrN-11450, and GrA-24154) are removed from this analysis due to contamination or misassociation. For further details of each of the 72 samples, please refer to Brindley (2007: Chapter 4). Three more measurements (GrN-15491, GrN-15492, and GrN-15493) were excluded from Brindley's analysis due to possible dietary offset, but are included in our analysis as these measurements do not appear to be anomalous when compared with the remainder. A total of 54 dates are therefore included in our analysis.

\subsection{Model}

Suppose we have $m$ number of date determinations. Let $x_{i}: i \in\{1,2, \ldots, m\}$ be the unknown calendar ages for these events. The trapezoid model of Karlsberg (2006) has 4 parameters: $\alpha, \beta, \gamma$, and $\delta$. Let $t_{\alpha}, t_{\beta}, t_{\gamma}$, and $t_{\delta}$ denote the calendar date associated with the trapezoid parameters. The model is defined mathematically as Karlsberg (2006):

$$
p_{\text {Trap }}\left(x_{i} \mid t_{\alpha}, t_{\beta}, t_{\gamma}, t_{\delta}\right)=h\left\{\begin{array}{cl}
0 & \text { for } x_{i}<t_{\alpha} \\
\frac{\left(x_{i}-t_{\alpha}\right)}{\left(t_{\beta}-t_{\alpha}\right)} & \text { for } t_{\alpha}<x_{i}<t_{\beta} \\
1 & \text { for } t_{\beta}<x_{i}<t_{\gamma} \\
\frac{t_{\delta}-x_{i}}{\left(t_{\delta}-t_{\gamma}\right)} & \text { for } t_{\gamma}<x_{i}<t_{\delta} \\
0 & \text { for } x_{i}>t_{\delta}
\end{array}\right.
$$


where $h$ is a constant, with values determined by the parameters

$$
h=\frac{2}{t_{\alpha}+t_{\delta}-t_{\beta}-t_{\gamma}}
$$

\subsection{Model Outputs}

Figure 1 summarizes the results from modeling the Irish bowl usage with a singe-phase trapezoid model (as specified in Karlsberg 2006) at 95.4\% confidence. The start and end of the Irish bowl usage are estimated to be in the ranges 2362-2168 BC and 1881-1720 BC ( $t_{\alpha}$ and $t_{\delta}$, respectively). This tradition is estimated to have continued for between 337 and $588 \mathrm{yr}$ and is calculated by subtracting $t_{\alpha}$ from $t_{\beta}$ after each iteration.

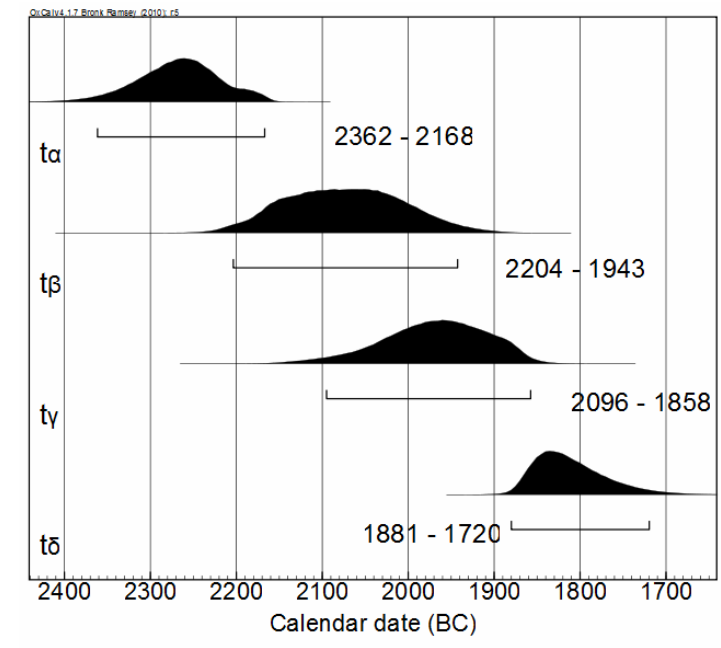

(a)

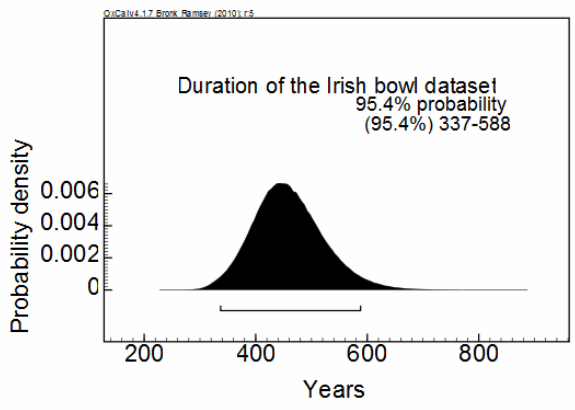

(b)

Figure 1 Probability density functions of the (a) trapezoid parameters and (b) timespan of the model at $95.4 \%$ confidence

How do the outputs differ from those of a uniform prior? Figure 2 summarizes the results from modeling with a single uniform phase at $95.4 \%$ confidence. In this case, the start and end of the Irish bowl usage are estimated to be in the ranges $2250-2151 \mathrm{BC}$ and $1902-1820 \mathrm{BC}$, respectively. This tradition is estimated to have continued for between 267 and $412 \mathrm{yr}$. The uniform parameters give more precise estimates than those of the trapezoid parameters. This is because the uniform parameters are estimating the onset and end of significant pottery use, whereas $t_{\alpha}$ and $t_{\delta}$ represent the very first and last use. In addition, the trapezoid model has more degrees of freedom and so it is inherently less constrained.

Even though the boundary parameters of the uniform-phase model give more precise temporal constraints for the Irish bowl data set, the trapezoid phase model is still preferred. This is because we do not have information to show that there is an abrupt change a priori. A trapezoid model better reflects the nature of the information that goes into the prior. 


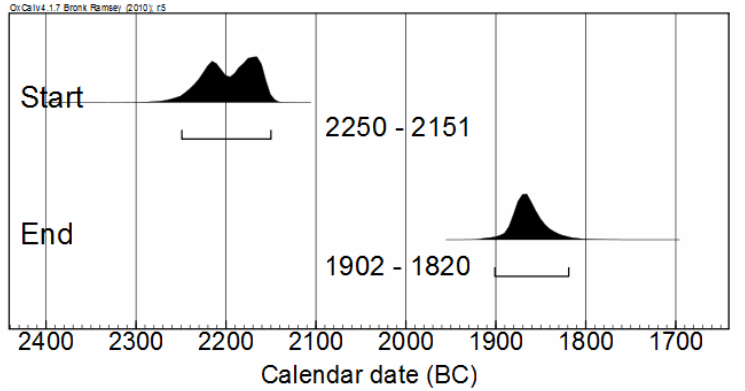

(a)

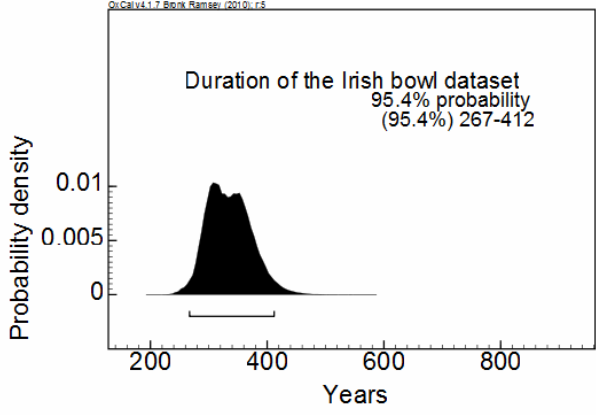

(b)

Figure 2 Probability density functions of the (a) uniform parameters and (b) timespan of the model at $95.4 \%$ confidence

\subsection{Alternative Parameterization}

Since the assumptions of the parameters of the trapezoid and uniform models are different, we propose an alternative parameterization of the trapezoid model with 2 boundary parameters $\left(t_{1}\right.$ and $t_{2}$ ) and 2 transition parameters $\left(d_{1}\right.$ and $\left.d_{2}\right)$, illustrated in Figure 3 . The boundaries with corresponding transitions make up Karlsberg's trapezoid parameters. The mathematics of the model and the overall prior remains the same despite the change of parameters. The trapezoid model becomes a uniform model when the length of transitions tend towards zero for the boundaries. The model is defined in this way because boundaries are commonly used in existing prior models (Buck et al. 1999; Bronk Ramsey 2009), and the trapezoid boundaries are comparable with those of the uniform prior. Midpoint boundaries will prove more useful in multiphase scenarios and will be discussed later.

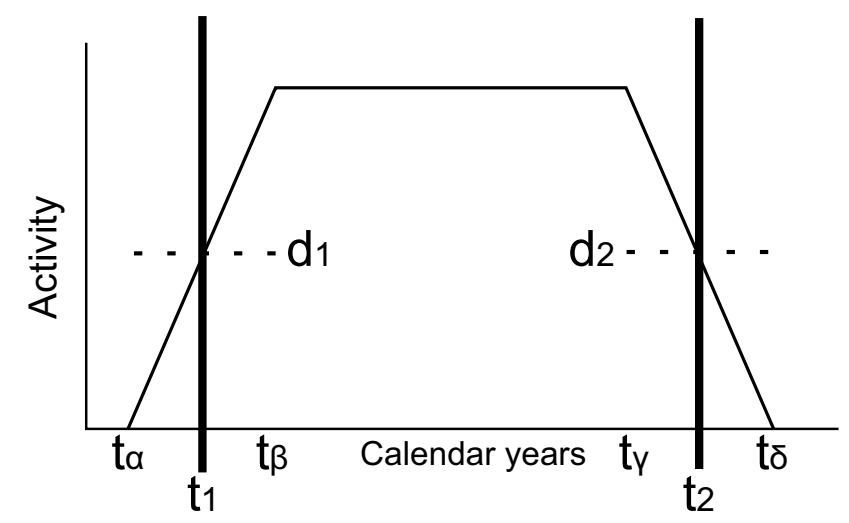

Figure 3 Schematic representation of the trapezoid prior model (Karlsberg 2006), where activity relates to usage, deposition, or production rate. The thick vertical lines are boundaries, $t_{1}$ and $t_{2}$; and the dotted horizontal lines are transitions, $d_{1}$ and $d_{2} \cdot t_{\alpha}, t_{\beta}, t_{\gamma}$, and $t_{\delta}$ are Karlsberg's parameters for the model, where $\alpha=t_{1}-d_{1} / 2, \beta=t_{1}+d_{1} / 2, \gamma=t_{2}-d_{2} / 2$, and $\delta=t_{2}+d_{2} / 2$.

\section{MODEL CONSTRAINTS}

Let $n$ be the number of phases. In a multiphase model with contiguous uniform phases, we have $t_{1}, \ldots, t_{n+1}$ boundaries. In a model with contiguous trapezoid phases, we also have $t_{1}, \ldots, t_{n+1}$ 
boundaries, with corresponding $d_{1}, \ldots, d_{n+1}$ transitions. A schematic diagram of a contiguous multiphase trapezoid model is shown in Figure 4. The overall timespan (S) of the model

$$
\mathrm{S}=t_{n+1}-t_{1}+\frac{d_{n+1}+d_{1}}{2}
$$

is made up of the total time for the transitions (D; gray areas in Figure 4) and the total time of the phase tops (T; white areas in Figure 4), where

$$
\mathrm{D}=\sum_{i=1}^{n+1} d_{i} \text { and } \mathrm{T}=t_{n+1}-t_{1}+\frac{d_{n+1}+d_{1}}{2}-\left(\sum_{i=1}^{n+1} d_{i}\right)
$$

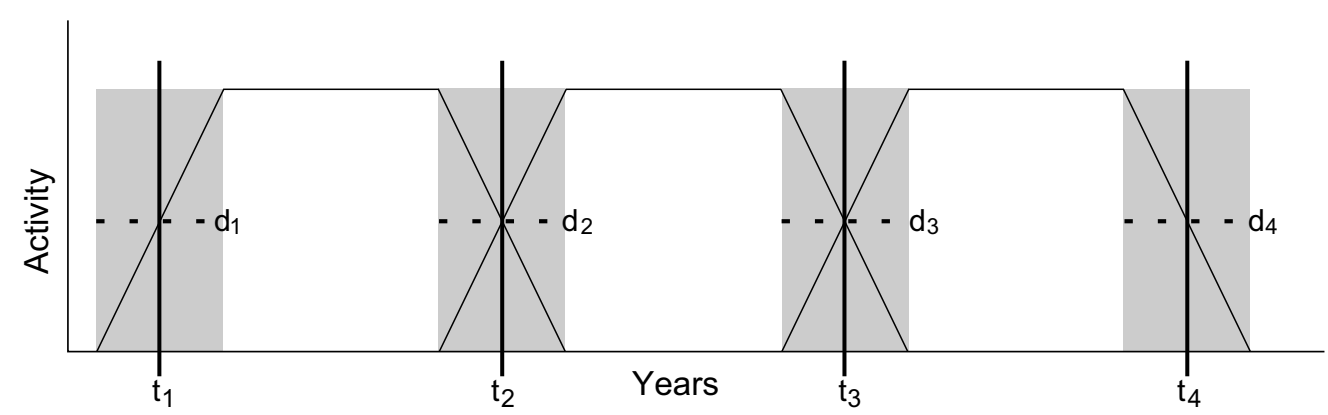

Figure 4 Schematic diagram showing the use of the trapezoid in a very specific contiguous multiphase trapezoid model. The thick vertical lines are the boundaries and the dotted horizontal lines are transitions. The transition parameters can be used to estimate the duration of transition between any 2 phases, highlighted in gray.

The constraints that exist in the current model are

$$
t_{L} \leq t_{1}-\frac{d_{1}}{2}<\ldots<t_{n+1}-\frac{d_{n+1}}{2}<t_{n+1}+\frac{d_{n+1}}{2} \leq t_{U}
$$

where $t_{L}$ and $t_{U}$ are terminus post quem and terminus ante quem, respectively. The calendar years are in the $\mathrm{AD}$ timescale, in which years $\mathrm{BC}$ are expressed with negative numbers.

Karlsberg (2006) suggests that the trapezoid model can vary in shape from uniform to triangular. We believe that this could be made more explicit. In the absence of ${ }^{14} \mathrm{C}$ data, the model parameters have a constant prior density (Nicholls and Jones 2001), i.e.

$$
p_{c p d}(t, \underline{d}) \infty 1
$$

for all $\underline{t}$ and $\underline{d}$ satisfying Equation 1. The sum of phase tops gives distributions as illustrated in Figure 5 . This weighting is caused by 3 factors:

1. The total timespan of the whole model has a bias proportional to $\mathrm{S}$.

2. The total timespan for transitions, of which there are $n+1$, has a bias proportional to $D^{n}$.

3. The total timespan for phase tops, of which there are $n$, has a bias proportional to $T^{n-1}$.

Under Equation 2, the marginal density for $\mathrm{T}$ is $\mathrm{T}^{n-1} \times \mathrm{D}^{n} \times \mathrm{S}$. Therefore, to obtain a uniform prior on $\mathrm{T}$, we propose using 


$$
p_{U T P}(t, \underline{d}) \infty \frac{\mathrm{T}^{1-n}}{\mathrm{D}^{n} \times \mathrm{S}}
$$

under which $\mathrm{T} \sim \mathrm{U}(0, \mathrm{~S})$ and $\mathrm{D} \sim \mathrm{U}(0, \mathrm{~S})$, by definition. We will call this the UTP, which stands for uniform transitions/tops prior, since it conditions the total timespan for transitions (D) and phase tops ( $\mathrm{T}$ ) to be uniform. The model becomes more robust and better reflects prior knowledge as expressed in Karlsberg (2006). The overall prior for the trapezoid parameters becomes

$$
p(t, \underline{d}) \infty p_{c p d}(t, \underline{d}) \times p_{U T P}(t, \underline{d})
$$

This is a similar idea to the uniform span prior in Nicholls and Jones (2001), which deals with multiple uniform phases.

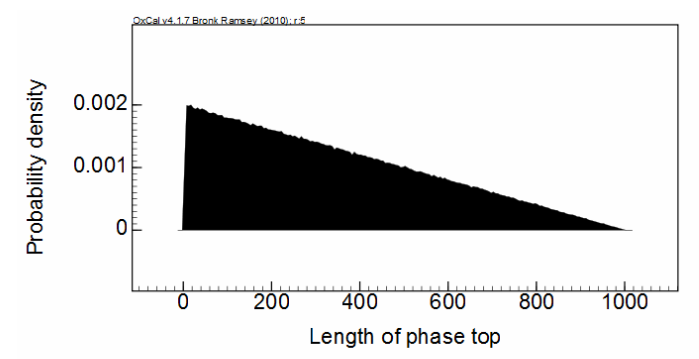

(a) Single phase

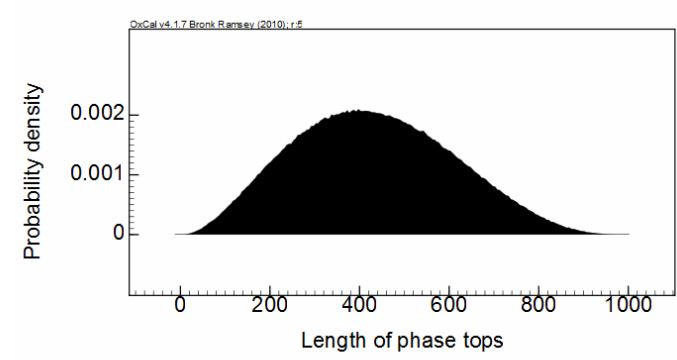

(c) 3 contiguous phases

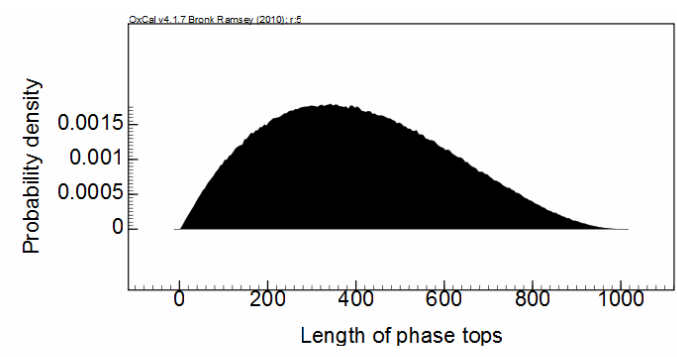

(b) 2 contiguous phases

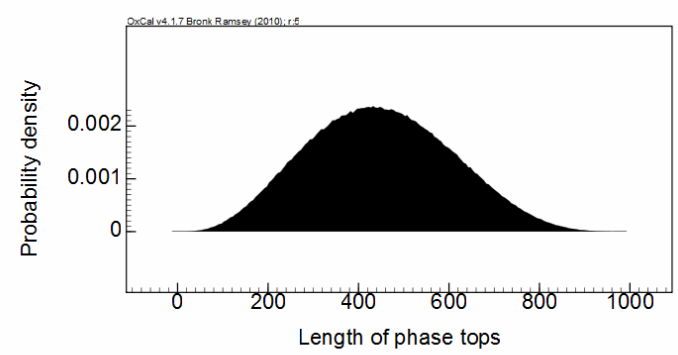

(d) 4 contiguous phases

Figure 5 Probability distributions of the sum of phase tops (T) under Equation 2 for up to 4 contiguous phases. The timespan (S) of the model is constrained to be 1000 .

\subsection{Model Limits}

If the model has limits, i.e. termini post quem and an ante quem, the timespan (R) between the 2 points is

$$
\mathrm{R}=t_{U}-t_{L}
$$

and the marginal density of the timespan of the trapezoid model has a bias towards $(R-S)$. Therefore, we propose a limits prior

$$
p\left(t,{ }_{-} \mid t_{L}, t_{U}\right) \infty(\mathrm{R}-\mathrm{S})^{-1}
$$


if the model is surrounded by events or fixed points. The overall prior for the trapezoid parameter becomes

$$
p(\underline{t}, \underline{d}) \infty p_{H}(t, \underline{d}) \times p_{U T P}(t, \underline{d}) \times p\left(t, \underline{d} \underline{d} \mid t_{L}, t_{U}\right)
$$

The trapezoid model can also be used in independent multiple-phase scenarios where the sequence of the parameters in each phase (indicated in square brackets) is specified if this can be identified from the archaeological evidence: for example, $t_{\beta}[n-1]<t_{\alpha}[n]<t_{\delta}[n-1]$ (notations from Figure 3). The limits prior would apply to such constraints, with the adjacent events to the model as terminus post quem and terminus ante quem.

\section{MODEL EVALUATION}

By constraining the timespan (S) of the trapezoid to be 1000, the effect of the UTP becomes apparent. In the absence of scientific dating information, $\mathrm{T} \sim \mathrm{U}(0, \mathrm{~S})$ is conditioned by Equation 4 , as illustrated in Figure 6. This means that the model is sensitive to the addition of the UTP.

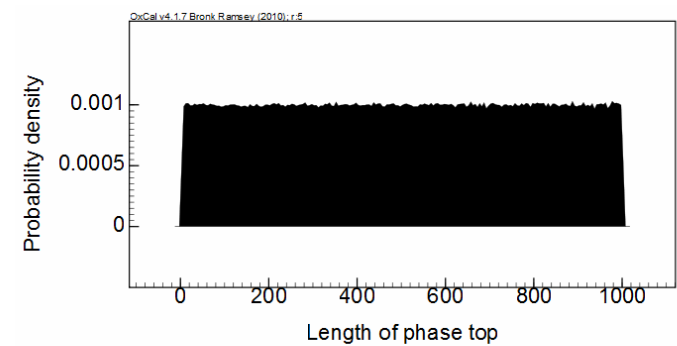

(a) Single phase

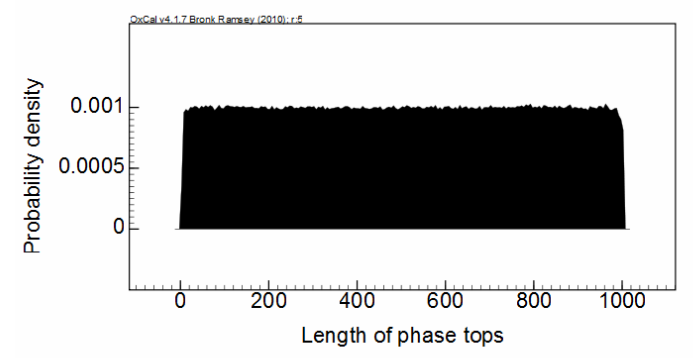

(c) 3 contiguous phases

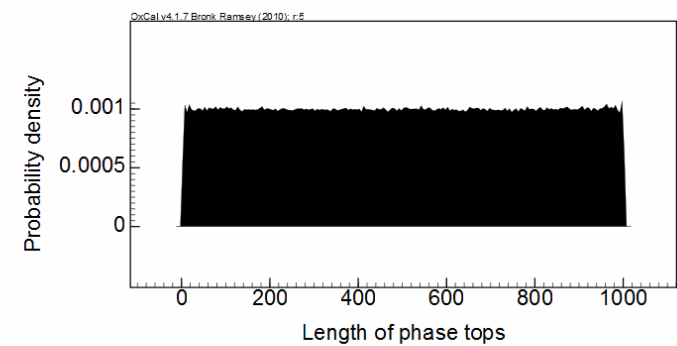

(b) 2 contiguous phases

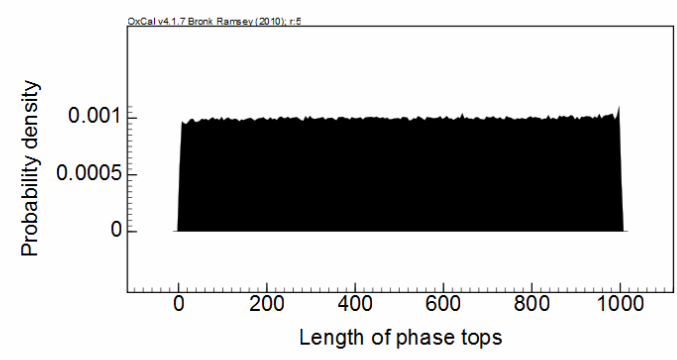

(d) 4 contiguous phases

Figure 6 Probability distributions of the sum of phase tops (T) under Equation 4 for up to 4 contiguous phases. The timespan (S) of the model is constrained to be 1000 .

A Markov chain Monte Carlo (MCMC) method is used to update the trapezoid parameters. Readers can refer to Gilks et al. (1996) for more information on constructing MCMC algorithms. A singlecomponent Metropolis-Hastings algorithm is used to implement the trapezoid throughout this paper. All the graphs are plotted in OxCal v 4 (Bronk Ramsey 2009), using the Prior function, after $2,000,000$ iterations and using a 5-yr bin size.

To test the model implementation, we simulated 2 scenarios with 3 contiguous phases, as illustrated in Figure 4: first, a scenario with no events and second with 10 undated events in each of the phases. 
The timespan (S) of the model is constrained to be 1000 , with $t_{1}-d_{1} / 2=0$ and $t_{4}+d_{4} / 2=1000$. The outputs are shown in Figures 7 and 8. The boundaries and transitions have the same distributions with and without undated events. This shows that the underlying assumptions of the trapezoid model (Figure 7) do not change with the addition of undated events (Figure 8), as would be expected from correct implementation of the MCMC algorithm.

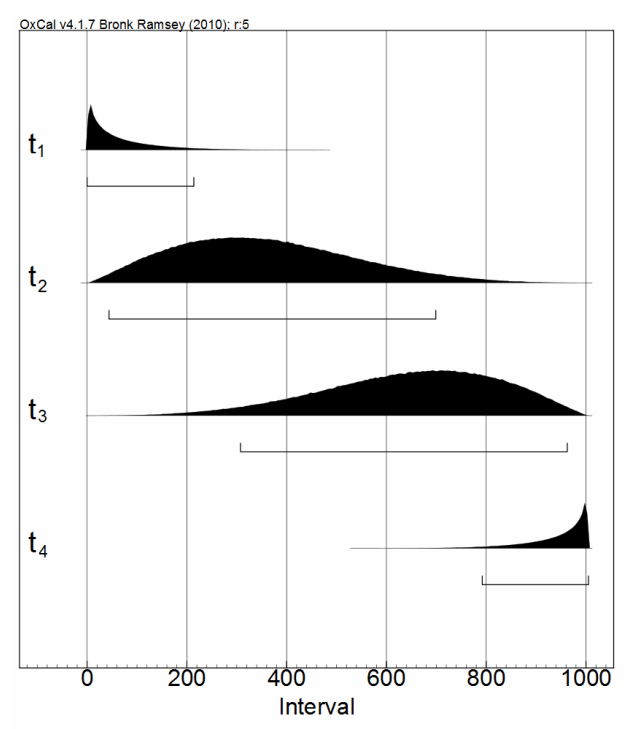

(a)

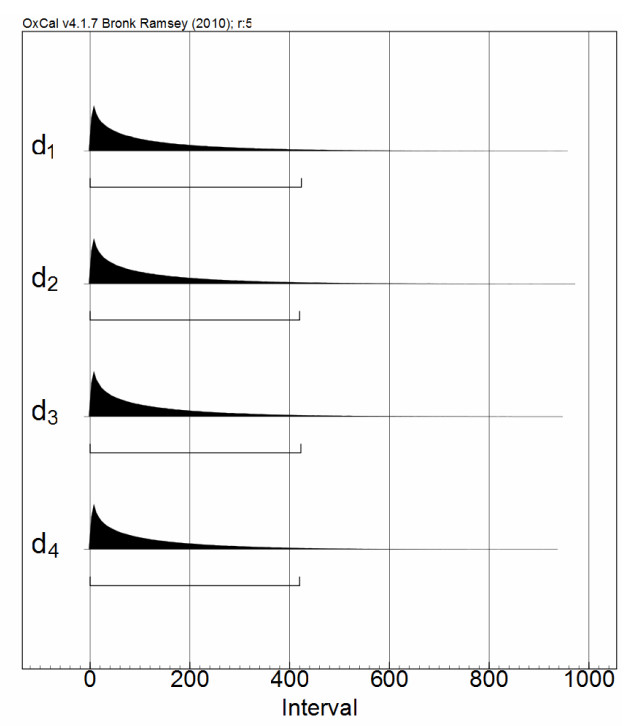

(b)

Figure 7 Marginal distributions of the (a) trapezoid boundaries and (b) transitions under Equation 4 for 3 contiguous phases with no events. The timespan (S) of the model is constrained to be 1000 .

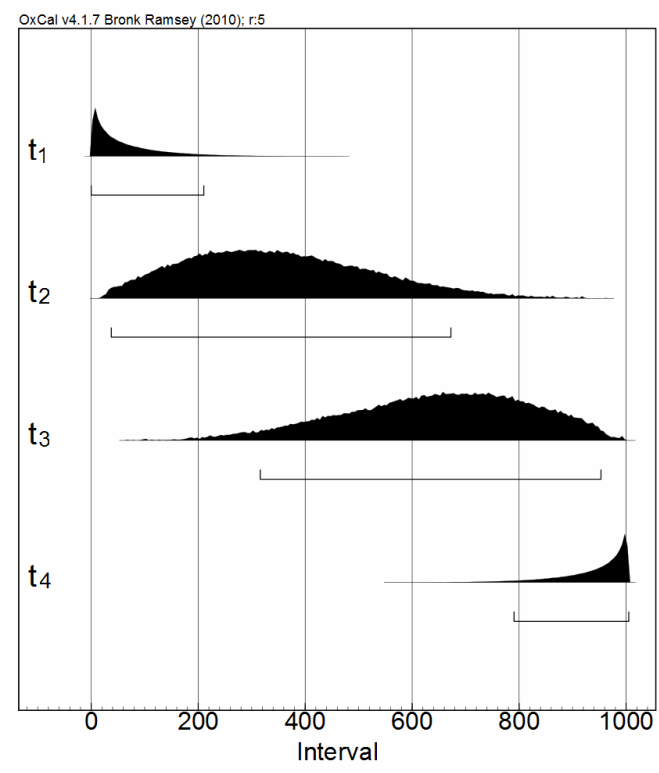

(a)

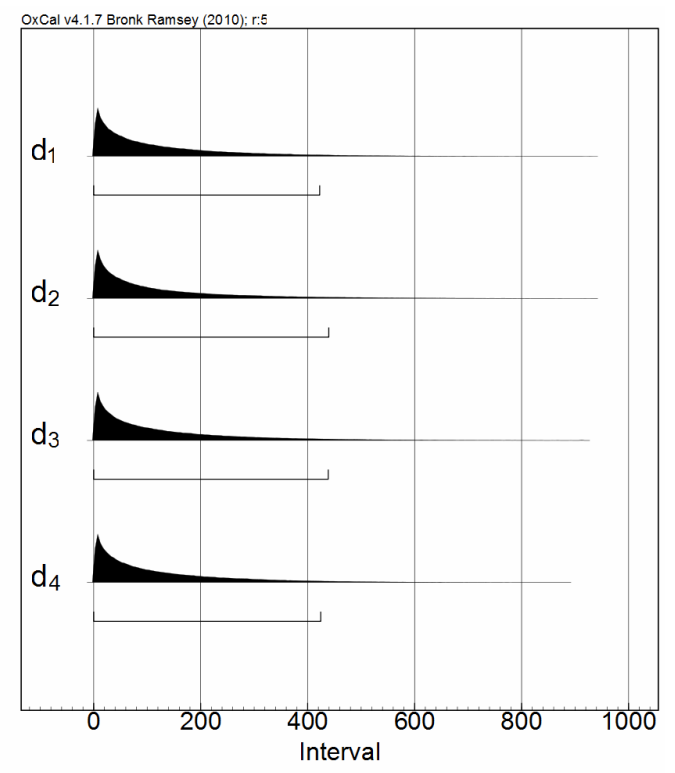

(b)

Figure 8 Marginal distributions of the (a) trapezoid boundaries and (b) transitions under Equation 4 for 3 contiguous phases with 10 events. The timespan (S) of the model is constrained to be 1000 . 
In the absence of scientific dating information, the transition parameters $(d)$ might take any values between $(0, D)$ with equal probability

$$
p(\underline{d})=\left\{\begin{array}{cl}
\frac{n}{D^{n}}(D-d)^{n-1} & \text { for } 0<d<D \\
0 & \text { otherwise }
\end{array}\right.
$$

and for $\mathrm{D} \sim \mathrm{U}(0, \mathrm{~S})$, conditioned by Equation 4, the marginal density of any transition parameters is represented by the choice

$$
p(\underline{d})=\frac{1}{S} \int_{d}^{S} \frac{n}{D^{n}}(D-d)^{n-1} d D
$$

This is illustrated in Figures 7 and 8 , as $d \rightarrow 0 ; p(d) \rightarrow \infty$. This means that short transition time is favored over long transition time. We also know that $d$ can never be zero and so the marginal distribution stays finite. The trapezoid model without this bias essentially rules out an overall abrupt transition model (the assumed scenario in a uniform-phase model).

Let us revisit the archaeological problem in Section 1 using the modified trapezoid prior. Figure 9 summarizes the results at $95.4 \%$ confidence. The start and end of the Irish bowl tradition are estimated to be in the ranges $2334-2159$ and $1887-1745 \mathrm{BC}\left(t_{\alpha}\right.$ and $\left.t_{\delta}\right)$, respectively. This tradition is estimated to have continued for between 305 and $545 \mathrm{yr}$.

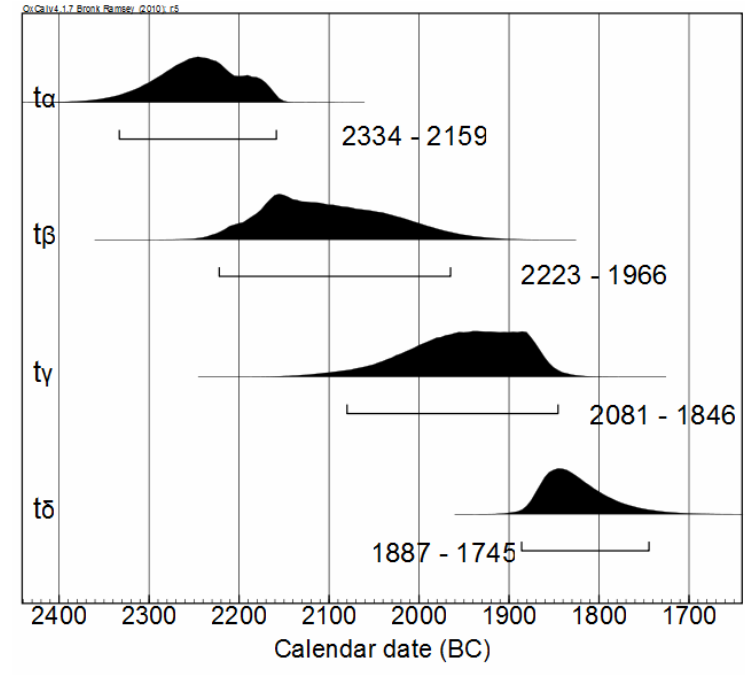

(a)

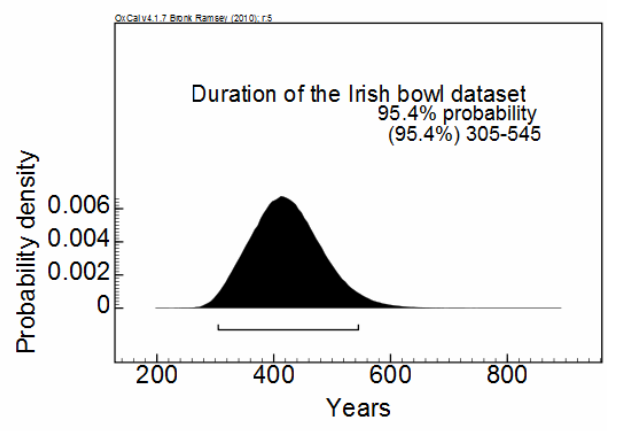

(b)

Figure 9 Probability density functions of the (a) trapezoid parameters and (b) timespan of the model, under Equation 4, at $95.4 \%$ confidence.

The new parameters (Figure 10) suggest that the middle of the start and end of the Irish bowl tradition happened in the ranges 2237-2111 and 1949-1834 BC $\left(t_{1}\right.$ and $\left.t_{2}\right)$, respectively. The difference between the boundaries are also calculated to be between 197 and $380 \mathrm{yr}$. It is more comparable to the timespan of the Irish bowl tradition modeled using the uniform prior model. The transi- 
tion for each of the boundaries $\left(d_{1}\right.$ and $\left.d_{2}\right)$ are estimated to be in the ranges $0-326$ and $0-289$ yr, respectively.

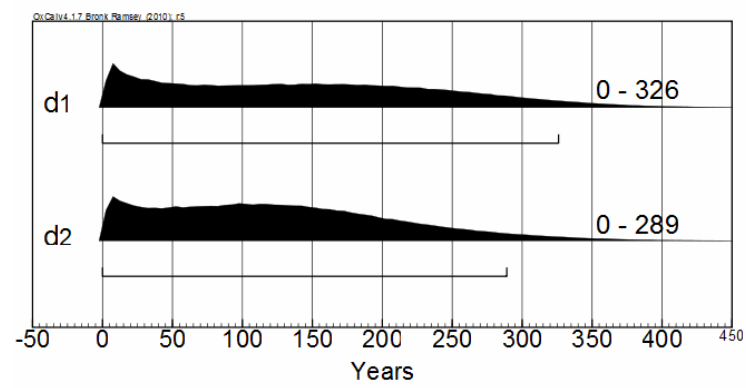

(a)

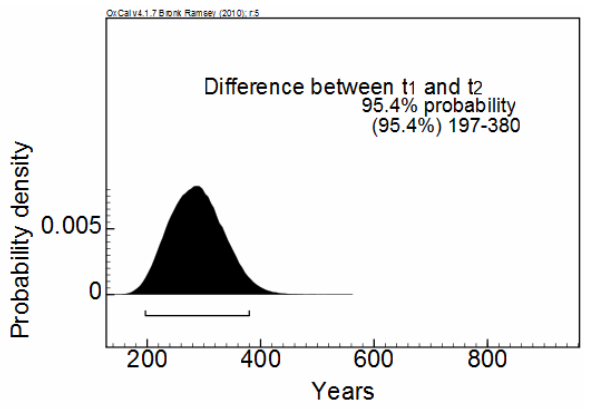

(b)

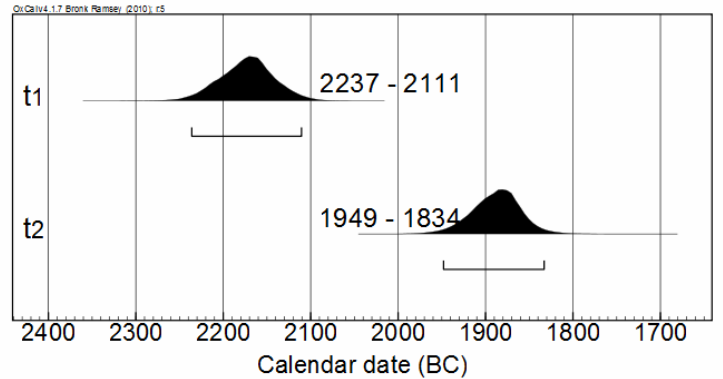

(c)

Figure 10 Probability density functions of the (a) new trapezoid parameters; (b) the difference between the boundary parameters $t_{1}$ and $t_{2}$; and (c) timespan of the model, under Equation 4 at $95.4 \%$ confidence.

Results from Figures 1 and 9 show that the UTP has a significant effect on the Irish bowl data set. It has reduced the timespan (S) of the model by 32-43 yr. The boundary estimates (Figure 10a) from the trapezoid model show a significant overlap $(80 \%)$ to those of a uniform model (Figure $2 \mathrm{a}$ ). The $95.4 \%$ intervals are shifted slightly in some cases because different underlying usage rates are assumed in both models. The estimates are more easily comparable with similar associated uncertainty.

\section{A MULTIPHASE MODEL}

Needham et al. (1997) obtained ${ }^{14} \mathrm{C}$ measurements from organic material in immediate contact with various types of bronze objects in an attempt to derive an independent chronology of British Bronze Age metalwork. Details of the archaeology and the treatment of the material are described in Needham et al. (1997). Thirty-eight dates were put into 5 phases: Acton and Taunton; Penard; Wilburton; Blackmoor; and Ewart Park. Two separate Bayesian models were constructed using the uniform prior: Model A, which assumes independent overlapping phases; and Model B, which assumes contiguous phases. Needham et al. (1997) concluded that the order of phases follows: Acton and Taunton; Penard; Wilburton; Blackmoor; and Ewart Park, which supports the relative Bronze Age chronology of the period.

In this section, we repeat the analysis using the trapezoid phase model to demonstrate its use in contiguous phases, and how the new parameters allow us to shed light on the duration of transition between adjacent phases. 


\section{Model A: Overlapping Trapezoid Phases}

Model A includes 5 independent overlapping trapezoid phases: Acton and Taunton; Penard; Wilburton; Blackmoor; and Ewart Park. This model assumes no ordering relationship between the 5 phases. Results of this model are summarized in Figure 11. The start and end dates of each phase are represented by the marginal distributions of $t_{\alpha}$ and $t_{\delta}$, respectively.

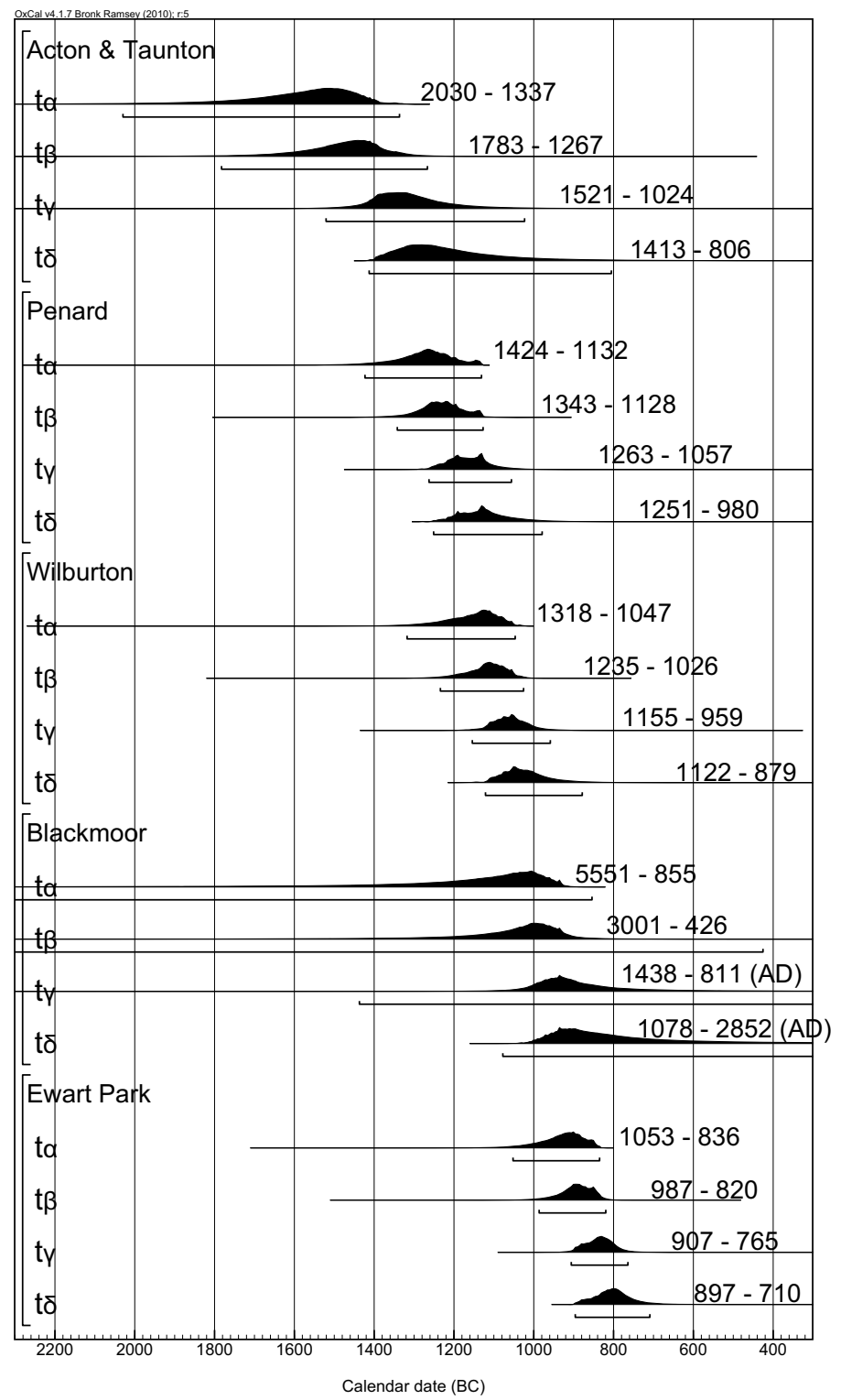

Figure 11 Probability density function of the trapezoid parameters for the Bronze Age phases, under Equation 4, at 95.4\% confidence. 
The degree of overlap between the rise and decline of phases are also investigated by calculating differences between the last 2 parameters of an earlier phase $\left(t_{\gamma}\right.$ and $\left.t_{\delta}\right)$ and the first 2 parameters of a later phase $\left(t_{\alpha}\right.$ and $\left.t_{\beta}\right)$. For example, in order to draw inference on the degree of overlap between the decline of Acton and Taunton and the rise of Penard, the marginal distributions representing the start and end of the rise of Penard $\left(t_{\alpha}\right.$ and $\left.t_{\beta}\right)$ are subtracted from the start and end of the decline of Acton and Taunton $\left(t_{\gamma}\right.$ and $\left.t_{\delta}\right)$, respectively. The differences between the parameters in Wilburton and Ewart park are also calculated due to the imprecision of the Blackmoor phase. The results are summarized in Figure 12. Table 1 summarizes the percentage of overlap between distributions.

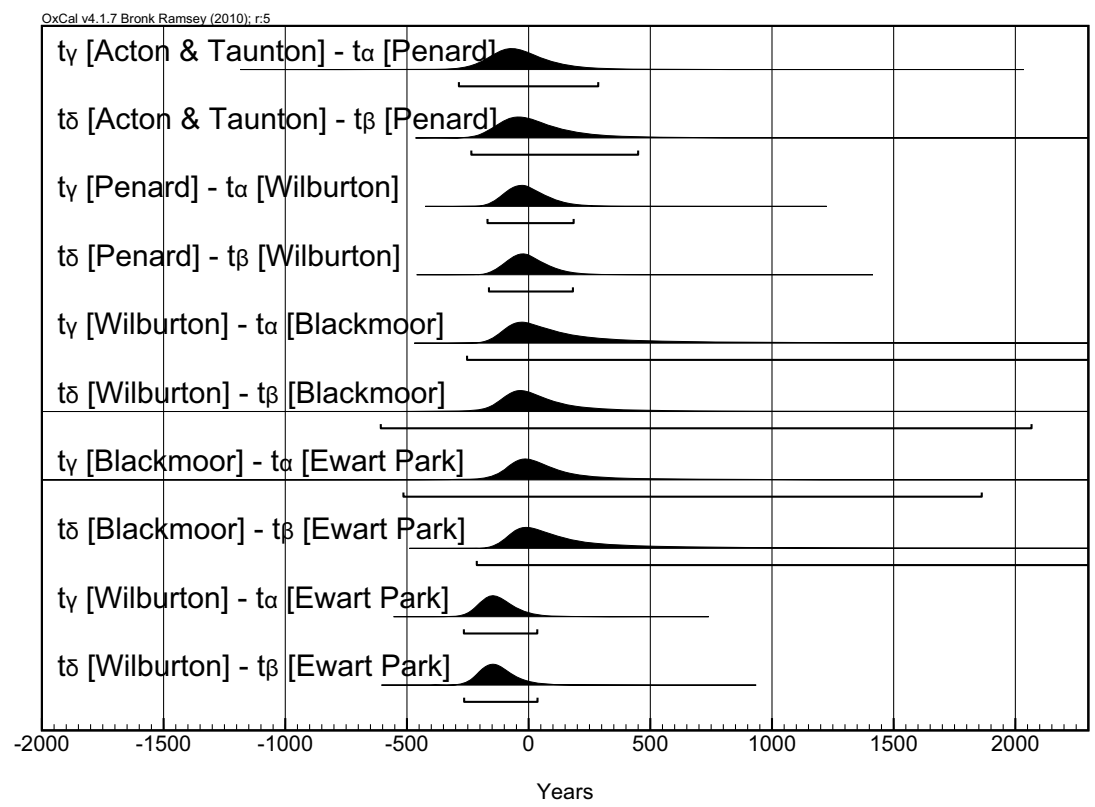

Figure 12 Estimates for the overlap between the rise and decline of adjacent phases at $95.4 \%$ confidence; negative values here indicate a gap in years between the specified parameters and positive values indicate an overlap.

Table 1 Estimates of the probability of overlap between the Bronze Age phases.

\begin{tabular}{ll|ll}
\hline \multicolumn{2}{c|}{ Phase } & \multicolumn{2}{c}{$\%$ overlap between distributions } \\
\hline$[\mathrm{A}]$ & {$[\mathrm{B}]$} & $t_{\gamma}[\mathrm{A}]$ and $t_{\alpha}[\mathrm{B}]$ & $t_{\delta}[\mathrm{A}]$ and $t_{\beta}[\mathrm{B}]$ \\
\hline Acton and Taunton & Penard & 36 & 52 \\
Penard & Wilburton & 44 & 46 \\
Wilburton & Blackmoor & 69 & 58 \\
Blackmoor & Ewart Park & 63 & 74 \\
Wilburton & Ewart Park & 6 & 7 \\
\hline
\end{tabular}

Results from this model suggest that the order of the Bronze Age subdivisions follows: Acton and Taunton; Penard; Wilburton; and Ewart Park, with a possible gap between the Wilburton and Ewart Park phases. The Blackmoor phase has very imprecise estimates due to the presence of only $3{ }^{14} \mathrm{C}$ dates in the phase. However, there is an overlap between Blackmoor and its adjacent phases. This leads us to believe that materials from the Blackmoor phase are chronologically intermediate between Wilburton and Ewart Park. This conclusion supports the relative chronology of O'Connor (1980) and also the analysis of Needham et al. (1997). 


\section{Model B: Contiguous Trapezoid Phases}

Model B includes 5 contiguous trapezoid phases: Acton and Taunton; Penard; Wilburton; Intermediate (characterized by Blackmoor); and Ewart Park (Figure 13a). This model assumes that the decline of an earlier phase overlaps entirely with the rise of a later, adjacent phase (as shown in Figure 4). Results of this model are summarized in Figure 13b.

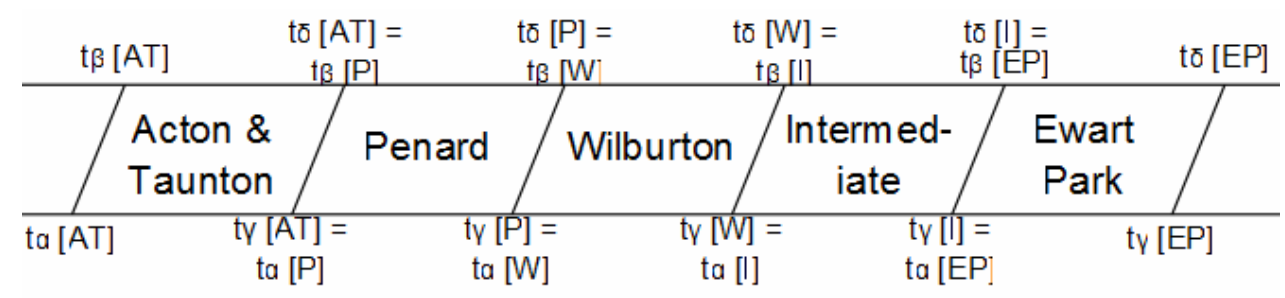

(a)

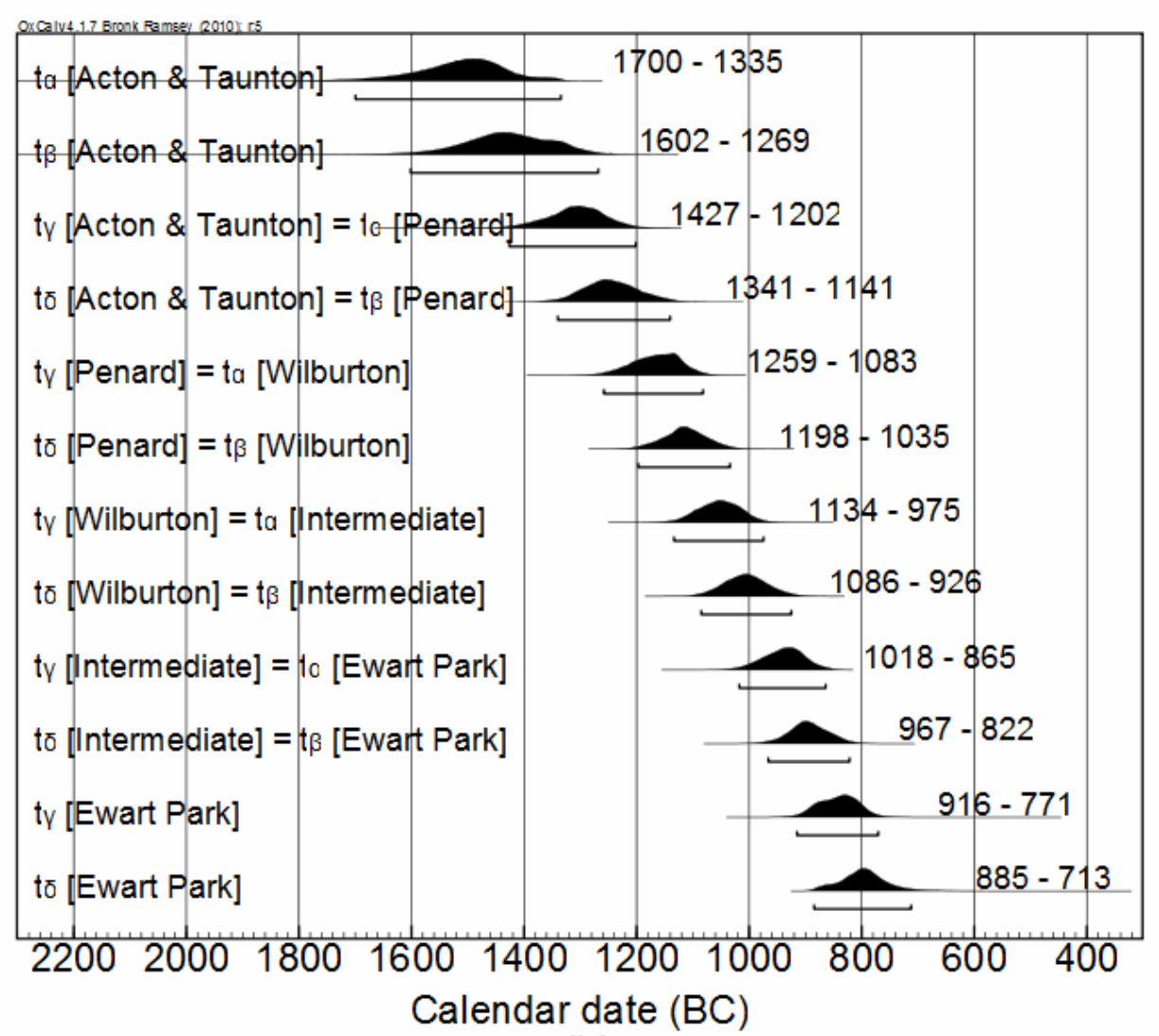

(b)

Figure 13 Model B: (a) the prior assumptions with the abbreviated phases in the square brackets; and (b) probability density functions of the trapezoid parameters for the Bronze Age phases, under Equation 4, at 95.4\% confidence. 
Both models (A and B) support the relative chronology of O'Connor (1980) that the subdivisions of British Bronze Age metalwork follow the order of Acton and Taunton; Penard; Wilburton; Intermediate (characterized by Blackmoor); and Ewart Park. The extra assumptions in Model B give a tighter overall chronology than the chronology given by Model A. The precision of the Intermediate phase has improved significantly.

The new parameters give estimates of the middle of the transitions between phases and the duration of each transition (Figure 14). The trapezoid model boundaries give similar estimates to those of the uniform model (Figure 15). This is an important finding as it confirms that the more robust trapezoid model backs up the analysis from the simpler, more rigid, uniform-phase model. The transition parameters give information on the duration of transitions between adjacent phases (Figure 14b). This information was previously not available with a uniform-phase prior.

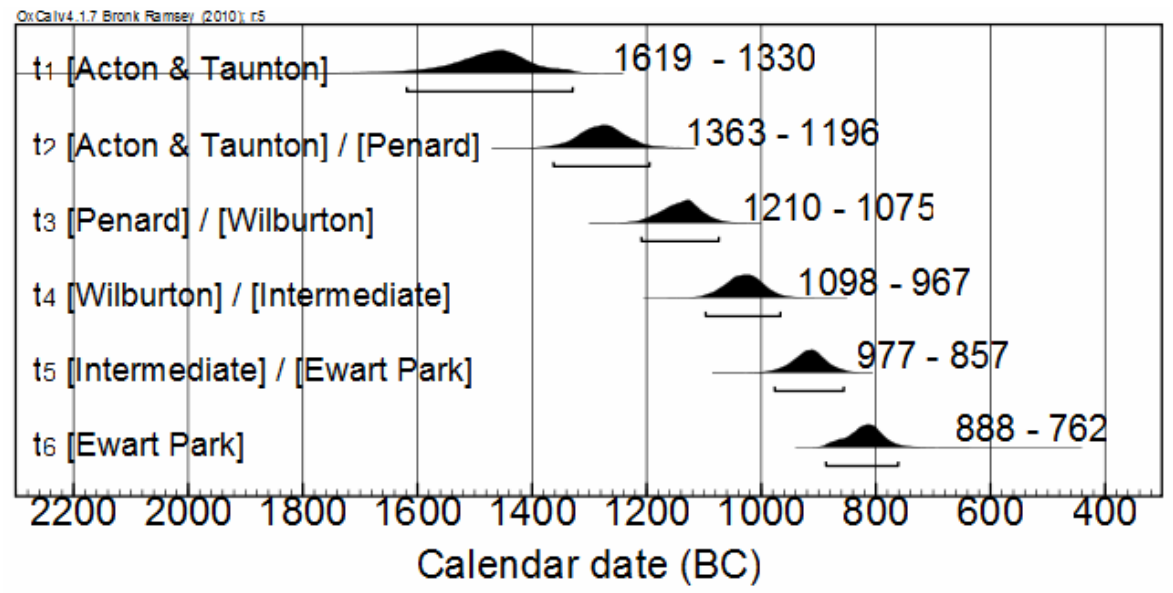

(a)

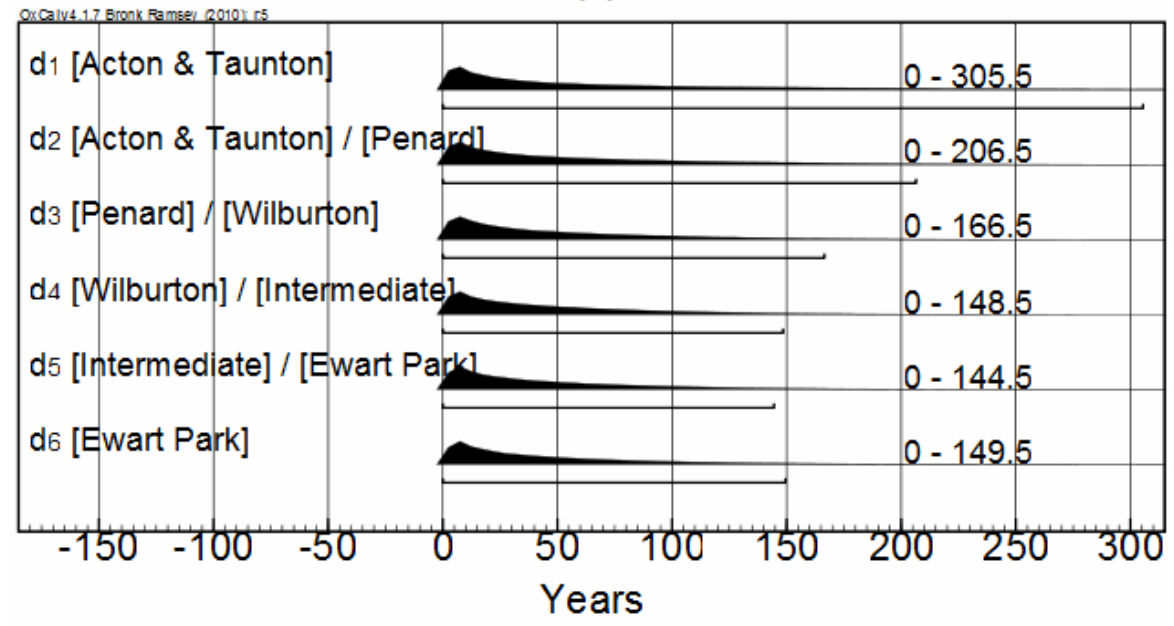

(b)

Figure 14 Probability density functions of the (a) trapezoid boundaries and (b) transitions for the Bronze Age phases, under Equation 4, at 95.4\% confidence. 


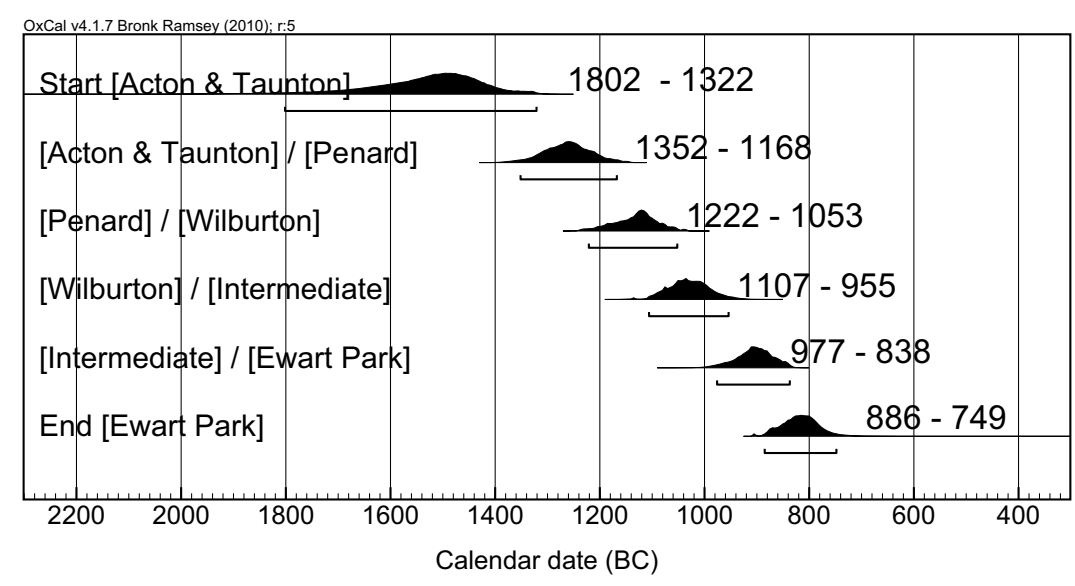

Figure 15 Probability density functions of the estimated calendar dates of transition between the Bronze Age phases at $95.4 \%$ confidence, modeled using contiguous uniform phases.

\section{SUMMARY}

In this paper, we analyzed 2 data sets with the trapezoid model. This model is more appropriate because a priori we assume the change in artifact types is non-abrupt. Using the trapezoid model better reflects the nature of the information that goes into the prior.

We also proposed 2 modifications to the trapezoid model: alternative parameterization and the addition of a UTP. Interestingly, the new parameters give comparable boundary estimates to the uniform model. One use of the trapezoidal model is for testing the robustness of models previously run under a uniform-phase prior where there was not good justification for the assumption of rapid phase transitions. Inference on the duration of transition between phases can also be drawn, which was not previously available with the uniform-phase model.

Addition of the noninformative UTP ensures model flexibility. The timespan of the phase tops (T) and the proportion of time spent in transitions (D) are uniform between the timespan S of the model, marginally. This poses a bias towards zero in individual transition parameters $(d)$. A model without this UTP rules out an overall abrupt model. Trapezoidal models for phases of activity are useful for situations where we expect activity to follow the pattern of a gradual increase, then a period of constant activity, and finally a gradual decrease (e.g. in the use of particular pottery types) and also in cases where we want a more robust prior than that provided by the uniform-phase model.

\section{ACKNOWLEDGMENTS}

The authors would like to acknowledge the important work of Angela Karlsberg, who first implemented the trapezoid model. Thanks are due to Alex Bayliss, Geoff Nicholls, Caitlin Buck, Richard Staff, Michael Dee, and 2 anonymous reviewers for suggestions. The research behind this paper was conducted in support of a NERC-funded project on Response of Humans to Abrupt Environmental Transitions (NE/E015670/1). 


\section{REFERENCES}

Bayliss A, O'Sullivan M. Forthcoming. Interpreting chronologies for the Mound of the Hostages, Tara and its contemporary Irish contexts. Tara Symposium, University College Dublin, 23-26 October 2009.

Blackwell PG, Buck CE. 2003. The Late Glacial human reoccupation of north-western Europe: new approaches to space-time modelling. Antiquity 77(296): $232-40$.

Blockley SPE, Lowe JJ, Walker MJC, Asioli A, Trincardi F, Coope GR, Donahue RE, Pollard AM. 2004. Bayesian analysis of radiocarbon chronologies: examples from the European Late-glacial. Journal of Quaternary Science 19(2):159-75.

Brainerd GW. 1951. The place of chronological ordering in archaeological analysis. American Antiquity 16(4): 301-13.

Brindley AL. 2007. The dating of food vessels and urns in Ireland. Bronze Age Studies. Volume 7. Galway: Department of Archaeology, National University of Ireland.

Bronk Ramsey C. 1995. Radiocarbon calibration and analysis of stratigraphy: the OxCal program. Radiocarbon 37(2):425-30.

Bronk Ramsey C. 2001. Development of the radiocarbon calibration program. Radiocarbon 43(2A):355-63.

Bronk Ramsey C. 2009. Bayesian analysis of radiocarbon dates. Radiocarbon 51(1):337-60.

Buck CE, Bard E. 2007. A calendar chronology for Pleistocene mammoth and horse extinction in North America based on Bayesian radiocarbon calibration. Quaternary Science Reviews 26(17-18):2031-5.

Buck CE, Litton CD, Smith AFM. 1992. Calibration of radiocarbon results pertaining to related archaeological events. Journal of Archaeological Science 19(5): 497-512.

Buck CE, Christen JA, James GN. 1999. BCal: an online Bayesian calibration tool. Internet Archaeology. URL: http://intarch.ac.uk/journal/issue7/buck/.

Gilks WR, Richardson S, Spiegelhalte DJ. 1996. Introducing Markov chain Monte Carlo. In: Gilks WR, Ri- chardson S, Spiegelhalter DJ, editors. Markov Chain Monte Carlo in Practice. London: Chapman \& Hall. p 1-20.

Housley RA, Gamble CS, Street M, Pettitt P. 1997. Radiocarbon evidence for the Lateglacial human recolonisation of Northern Europe. Proceedings of the Prehistory Society 63:25-54.

Karlsberg AJ. 2006. Flexible Bayesian methods for archaeological dating [PhD thesis]. Sheffield: University of Sheffield.

Needham S, Bronk Ramsey C, Coombs D, Cartright C, Pettitt PB. 1997. An independent chronology for British Bronze Age metalwork: the results of the Oxford Radiocarbon Accelerator Programme. Archaeological Journal 154:55-107.

Nicholls G, Jones M. 2001. Radiocarbon dating with temporal order constraints. Applied Statistics 50(4):50321.

O'Connor B. 1980. Cross-channel Relations in the Later Bronze Age. Volumes 91 of International Series 91. British Archaeological Reports. Oxford: Archaeopress.

Reimer PJ, Baillie MGL, Bard E, Bayliss A, Beck JW, Blackwell PG, Bronk Ramsey C, Buck CE, Burr GS, Edwards RL, Friedrich M, Grootes PM, Guilderson TP, Hajdas I, Heaton TJ, Hogg AG, Hughen KA, Kaiser KF, Kromer B, McCormac FG, Manning SW, Reimer RW, Richards DA, Southon JR, Talamo S, Turney CSM, van der Plicht J, Weyhenmeyer CE. 2009. IntCa109 and Marine09 radiocarbon age calibration curves, 0-50,000 years cal BP. Radiocarbon 51(4): 1111-50.

Van Strydonck M, De Moor A, Bénazeth D. $2004 .{ }^{14} \mathrm{C}$ dating compared to art historical dating of Roman and Coptic textiles from Egypt. Radiocarbon 46(1):23144.

Zeidler JA, Buck CE, Litton CD. 1998. Integrating of archaeological phase information and radiocarbon results from the Jama River Valley, Ecuador: a Bayesian approach. Latin American Antiquity 9(2):160-79. 